\title{
The physico-chemical history of Falling Evaporating Bodies around $\beta$ Pictoris: investigating the presence of volatiles
}

\author{
C. Karmann ${ }^{1}$, H. Beust ${ }^{1}$, and J. Klinger ${ }^{2}$ \\ 1 Laboratoire d'Astrophysique de l'Observatoire de Grenoble, Université J. Fourier, BP 53, \\ 38041 Grenoble Cedex 9, France \\ 2 Laboratoire de Planetologie de Grenoble, Université J. Fourier, BP 53, 38041 Grenoble Cedex 9, France
}

Received 22 December 2000 / Accepted 12 March 2001

\begin{abstract}
Transient spectral absorption events have been monitored for many years toward the star $\beta$ Pictoris and have been interpreted as resulting from the transit across the line of sight of evaporating comet-sized bodies (Falling Evaporating Bodies, or FEBs). The model shows that these bodies come from circular orbits at $\gtrsim 4 \mathrm{AU}$, becoming star-grazers due to planetary perturbations. The physics of the evaporation of those bodies is widely influenced by their physico-chemical properties, especially the presence of volatile matter. We investigate here this question from a modeling point of view, adapting for that case the models designed for solar comets. We simulate the physico-chemical evolution of the FEB progenitors on circular orbits for a time comparable to the supposed age of $\beta \mathrm{Pic}$, and constrain the quantity of volatiles present in them in relationship with its age and to the semi-major axis of the orbits. We also constrain this semi-major axis by investigating its influence on the dynamical model of FEB generation by planetary perturbations, and show that it is probably less than $\sim 10 \mathrm{AU}$. Finally, we show that demanding the FEB progenitors to be icy in a major part of their volume at those distances is probably an unrealistic constraint, and that they more likely look like asteroids with perhaps a small icy nucleus rather than fully icy comets. This result leads to a revision of the FEB evaporation model that has been assumed up to now.
\end{abstract}

Key words. stars: $\beta$ Pic - methods: numerical - comets: general - stars: circumstellar matter planetary systems: formation

\section{Introduction}

\section{1. $\beta$ Pictoris}

The dusty and gaseous disk surrounding the southern star $\beta$ Pictoris ( $\beta$ Pic) (Smith \& Terrile 1984) is considered today as a key example of a young planetary system in its early dynamical history, with an age of typically a few $10^{7} \mathrm{yr}$. This kind of system is characterized by a large amount of dust diffusing the stellar light but an optically thin disk, as any primordial optically thick disk is thought to have been removed at this age. It is known today that the dust viewed is not a remnant of this primordial disk, but rather is second generation material continuously replenished from inside the disk by larger, planetesimal-like bodies (see reviews by Artymowicz 1997; Vidal-Madjar et al. 1998; Lagrange et al. 2000).

The age of $\beta$ Pic itself has been subject to controversy in the past years. $\beta$ Pic is a young main sequence A5 type star, and its age was initially estimated to be at least

Send offprint requests to: C. Karmann, e-mail: ckarmann@obs.ujf-grenoble.fr
$10^{8}$ yr. Paresce (1991), tentatively attributing the star's under-luminosity to an effect of its low metallicity with respect to the sun, proposed an age of $2 \times 10^{8} \mathrm{yr}$. Conversely, Lanz et al. (1995) attributed the under-luminosity to extinction, proposing that $\beta$ Pic was actually a pre-mainsequence star not older than $10^{7} \mathrm{yr}$. Brunini \& Benvenuto (1996) and Artymowicz (1997) concluded that any age between $10^{7}$ and $10^{8} \mathrm{yr}$ could fit the HR diagram constraints. Meanwhile, the stellar parameters of the star were re-measured by Crifo et al. (1997), which showed that the apparent under-luminosity of the star was formerly due to a bad estimate of the distance of the star. Vidal-Madjar et al. (1998), noting that $\beta$ Pic does not lie in the vicinity of any star-forming region, claimed that this was not compatible with an age less than $3 \times 10^{7} \mathrm{yr}$, but Barrado y Navascués et al. (1999) analyzed carefully the Hipparcos data from a moving stellar group tentatively associated to $\beta$ Pic, concluding that the group was real, and proposed an age of $20 \pm 10 \times 10^{6} \mathrm{yr}$, by estimation of the age of three $\mathrm{M}$ dwarfs associated with the group. In conclusion, it is still impossible to give a more accurate range than $2 \times 10^{7}-10^{8} \mathrm{yr}$. 


\subsection{Falling Evaporating Bodies and their chemical composition}

Independent of disk imaging, transient spectral absorption events occurring in metallic lines have been monitored for several years around $\beta$ Pictoris (Vidal-Madjar et al. 1994; Lagrange et al. 1996). These events tend to vary on very short time-scales (days or even hours), and have been successfully interpreted as resulting from the transit across the line of sight of evaporating star-grazing comet-sized bodies (Falling Evaporating Bodies, or FEBs). A model has been developed which reproduces the observed events (Beust et al. 1990, 1996, 1998) with many of their characteristics.

In spite of its ability to correctly explain the features observed, this model is very sensitive to supposed physico-chemical properties of the evaporating bodies. Every change in chemical composition has an effect on the simulated spectral absorptions, both in terms of compared strengths of variable lines expected, and of dynamics of the corresponding species once escaped from the FEBs. In previous models, the assumption was that FEBs share the same chemical composition with Solar comets, i.e., refractory compounds having Solar composition relative to each other, and a strong depletion in volatile species.

Direct chemical composition measurements on the variable features attributed to FEBs is not straightforward, as the depths of these components do not depend only on the abundance of the corresponding species, but also on the filling factor of the absorbing clouds in front of the stellar disk. It has been showed by comparison of variable features occurring in doublet lines that the absorbing material was mostly clumpy (Vidal-Madjar et al. 1994; Lagrange et al. 1996). Modeling (Beust et al. 1996) showed that the projected size of the ionic clouds escaping from the FEBs onto the stellar surface actually depends on the dynamics of the species they contain; moreover, this dynamic tends to differ from a given element to another (due to the variations in the radiation pressure). Consequently, measuring the chemical composition of FEBs from their spectral signatures cannot be performed independent of dedicated modeling. In this context, it has thus not been possible to heavily constrain the chemical composition of FEBs. Concerning refractory species, all that can be stated today is that Solar (i.e., cometary) composition is compatible with the observations and the models within one order of magnitude. Conversely, the composition of the stable circumstellar gas is better constrained (Lagrange et al. 1998), with the result that refractory species indeed have Solar relative composition. If we assume this stable gas also to be linked to FEBs (because it needs to be permanently refilled; see Lagrange et al. 1998), then we may suppose that this also applies to the chemical composition of the FEBs themselves.

A remaining key issue is nevertheless the presence of volatiles in the FEBs. This issue is much more difficult to constrain, as most of the spectral variations observed are in the visible/UV spectral range and concern metal- lic ions. It nevertheless has an important effect on the resulting components observed in metallic species: when metallic ions sublimate from the body, they automatically disperse into space. In order to produce significant absorption features such as those observed, they must be retained for a while in the vicinity of the FEB to make a sufficiently dense cloud, before they are blown away by radiation pressure. In the models developed thus far, volatile compounds (dissociated water, CO) are assumed to be released by the FEBs with cometary abundance, i.e. with an abundance roughly equal to that of the refractory compounds. In this case, as the volatile species mostly do not feel an intense radiation pressure from the star, they efficiently retain the metallic ions in the vicinity of the nucleus.

Volatile species can be produced by the sublimation of ice usually present in comet-like bodies. The assumption that volatiles are actually abundant in FEB progenitors needs however to be questioned in relation to the dynamical model that is currently invoked as accounting for the origin for these numerous star-grazers (Beust \& Morbidelli 1996, 2000): a FEB progenitor is a body initially trapped in $3: 1$ or $4: 1$ mean-motion resonance with a Jovian-like planet orbiting the star on a moderately eccentric orbit $\left(0.05 \lesssim e^{\prime} \lesssim 0.1\right)$. Secular resonant perturbations by the planet are then able to bring the orbital eccentricity of the bodies very close to 1 (without changing their semimajor axis), putting them on star-grazing orbits within $\sim 10^{4}$ planetary revolutions. This model is by far the best match with the observed orbital properties of FEBs when they reach the star-grazer state. Depending on the orbital semi-major axis of the planet, the FEB progenitors are thus bodies originating from circular orbits at typically a few AUs from the star (4-16 AU). Consequently, the FEB progenitors are supposed to have been orbiting the star at such distances since the formation of the system, before they start a rapid eccentricity increase which brings them to the FEB state. The FEB progenitors have therefore been exposed to the stellar radiation flux of $\beta$ Pic at such distances for more than $2 \times 10^{7} \mathrm{yr}$. In this context, it is not sure whether ices, even embedded in a refractory matrix, would survive sublimation for such a long time. Recall that the luminosity of $\beta$ Pic is 8.7 times that of the Sun (Crifo et al. 1997). From this strict point of view, FEBs orbiting $\beta$ Pic between 4 and 16 AU receive the same radiation flux as bodies orbiting the Sun between 1.35 and 5.42 AU. At the present age of the Solar System, kilometer-sized bodies orbiting the Sun in this region (i.e., asteroids) are mainly made of refractory material. However, the Solar System is significantly older than $\beta$ Pic, so that it is still impossible to make definitive statements about $\beta$ Pic FEB progenitors on the basis of this simple comparison.

It is then of interest to investigate this question in more detail in the context of the stellar environment of $\beta$ Pic, and more precisely, to state in which conditions and at which stellar distances ices are likely to survive alone or embedded in rocky bodies. This is the purpose of the present paper. In this work, we scale models that have been used for Solar comet modeling to the $\beta$ Pic case. 
In Sect. 2, we describe the method used. In Sect. 3, we first apply it to purely icy spheres, and in Sect. 4 we present the results concerning a more realistic body made of a mixture of ices and dust. As expected, the result strongly depends on the stellar distance from which the FEB progenitors are supposed to originate. In Sect. 5, we then describe the constraints on this distance that can be derived from independent dynamical considerations concerning the origin of FEBs. In Sect. 6, we present the implications of this study for our present knowledge of the FEB evaporation process, and our general conclusions are presented in Sect. 7.

\section{The method}

\subsection{General assumptions}

Knowing that this work is exploratory, we tried to avoid any oversophistication. We thus adopted the approach developed by Espinasse et al. $(1989,1991)$ rather than more up-to-date concepts which have been developed since that time (see for example Tancredi et al. 1994; Enzian et al. 1997).

We model an FEB progenitor as a porous sphere of matter, mainly composed of ice and silicates. We will first consider, for comparison purposes, a porous icy sphere, and then consider a mixture of ice and dust in the form of $\mathrm{SiO}_{2}$.

When their periastron reaches star-grazing values, the FEBs evaporate volatiles and refractory material. These refractory species are actually the ones we observe as spectral variations. The dynamical process that brings the bodies into such orbits is gradual. The FEB progenitors we want to model here are thought to originate from nearly circular orbits further away from the star, typically between 4 and $16 \mathrm{AU}$. At such distances, refractory material does not evaporate (the limit for dust evaporation is $\sim 0.4$ AU; see Beust et al. 1998). Consequently, in our approach, we need only consider the process of evaporation. Dust is thus considered as inert matter, which does not react in any way and whose thermodynamic properties are taken from the literature. The FEB is assumed to orbit the star on a circular orbit at a few AU, so that it always receives the same radiation flux from the star.

As long as this assumption is valid, there is no need to consider that the sublimation rate is affected by other phenomena, such as fragmentation within the object. Of course, when it later becomes a star-grazer, these processes can play a significant role (Tambovtseva \& Shestakova 1999), especially in a hotter environment than the Sun, as is the case here (Grinin et al. 1996). This is beyond the scope of the present study.

We also make the assumption that the environment of the progenitor does not change during its history, especially the brightness of the star. This is actually a fairly crude approximation because $\beta$ Pictoris is a young star and has undergone the correspondingly tumultuous history of a young A type star. Up to time-scales of a few $10^{6} \mathrm{yr}$, the approximation is nevertheless justified.
However, this approximation should not drastically affect the first order results we derive below.

The FEB progenitor is supposed to be a fast rotator, so that we can consider a spherical thermal symmetry, without having to include a day/night cycle. The fast rotator concept is not very realistic, but has the advantage of giving a lower limit to the sublimation ratio of matter and thus an upper limit to the lifetime of the body, which is what we are seeking.

\subsection{Physical processes}

As mentioned above, we need only consider ice evaporation. Dust interacts with ice only through its thermodynamical properties (heat conduction, heat capacity).

Initially, the material is considered to be in thermodynamical equilibrium. Each temperature rise (or fall) induces sublimation (or condensation). Kinetic gas theory gives, for the statistical sublimation/condensation rate of a species $i$ :

$Q_{\epsilon, i}=\Sigma \cdot \alpha \frac{p_{i}^{\mathrm{s}}-p_{i}}{\sqrt{2 \pi M_{i} R T}}$ moles s ${ }^{-1} \mathrm{~m}^{-3}$,

where $Q_{\epsilon, i}$ is the sublimation rate of the species within a unit volume, $T$ is the temperature, which is equal in the solid phase and in the gas, $R$ is the universal gas constant and $M_{i}$ is the molar mass of species $i . p_{i}^{\mathrm{s}}$ stands for the saturated vapour pressure and $p_{i}$ for the partial pressure. $\Sigma$ is the volume-specific surface area. $\alpha$ is the sticking (or condensation) coefficient.

If enough ice is present, the characteristic time-scale of sublimation is much shorter than those of heat and gas diffusion (Tancredi et al. 1994). Hence, in that case, the gas is close to saturation and so automatically compensates for the local loss or gain of gas due to gas diffusion towards the surface. We can then take a sublimation/recondensation rate equal to (Espinasse et al. 1991):

$Q_{\epsilon i}=\frac{N_{\text {gas }}-N_{\mathrm{sat}}(T)}{\delta t}=\frac{p_{i}-p_{i}^{\mathrm{s}}(T)}{R T \delta t} \cdot \Pi$ moles s ${ }^{-1} \mathrm{~m}^{-3}$

where $\delta t$ is the time step of our simulation, and $\Pi$ the porosity.

At the surface, the gas which is the product of sublimation on the surface and of diffusion from the interior of the body automatically disperses into space. Consequently, we never reach an equilibrium between sublimation and recondensation, and the sublimation rate is derived from the statistical sublimation rate, with $p_{i}=0$. This approximation is in fact very good. In all the simulations described below, we independently computed the surface pressure from our results. We always obtain a pressure that is less than saturated vapour pressure by several orders of magnitude.

Taking into account the typical equilibrium temperature obtained (more than $200 \mathrm{~K}$ for blackbodies of albedo 0.1 and emissivity 0.9 which are closer than $16 \mathrm{AU})$, ice can be safely considered as crystalline. 


\subsection{The heat equation}

With our assumption of spherical symmetry, the heat transfer equation takes the form:

$\rho(r) C(r) \frac{\partial T}{\partial t}=\nabla(K(r, T) \cdot \nabla T)+Q_{\epsilon}^{\prime}$,

where $r$ is the distance to the center of the body and $T$ the temperature. $C$ stands for the heat capacity, $\rho$ for the density, $K$ for the thermal conductivity and $Q_{\epsilon}^{\prime}$ for the source term which describes thermodynamical phenomena encountered (sublimation and recondensation, sensitive heat).

Thermal conductivity is computed by considering a parallel network. The thermal conductivity of compact matter is:

$K^{c}=\frac{\rho_{\mathrm{H}_{2} \mathrm{O}} K_{\mathrm{H}_{2} \mathrm{O}}+\rho_{\text {dust }} K_{\text {dust }}}{\rho_{\mathrm{H}_{2} \mathrm{O}}+\rho_{\text {dust }}}$,

where $K_{\mathrm{H}_{2} \mathrm{O}}$ and $K_{\text {dust }}$ are respectively the ice and dust thermal conductivities by unit of mass.

Porosity also has a strong effect on the thermal conductivity. The real thermal conductivity of porous matter is derived from the conductivity computed above by a Russel equation where the gas conductivity is considered null:

$K=\frac{1-\Pi^{2 / 3}}{1-\Pi^{2 / 3}+\Pi} \cdot K^{\mathrm{c}}$,

where $\Pi$ stands for the porosity. This equation, which was used in Espinasse et al. (1989, 1991), does not give a very good value for the conduction, as it does not take into account the structural parameters of the matter. In fact, measurements performed on porous icy matter by Spohn et al. (1989) show that the real conduction is more than ten times lower than the conduction predicted by the Russel equation. However, as the structure of cometary matter is not well known, we keep this formula, multiplying it by a constant Hertz factor $h(h \leq 1)$. Simulations performed later showed that changing this factor does not drastically modify the results.

Another contribution to conduction is the sensitive heat, i.e., the heat transported by the gas which diffuses throughout the porous matrix. This effect is computed as a term of $Q_{\epsilon}^{\prime}$, by making the assumption that the gas is always thermalized, i.e., it has the same temperature as the matter surrounding it.

Boundary conditions of this equation are, at the center of the body $(r=0)$ :

$\frac{\partial T}{\partial r}=0$.

At the surface, we balance the energy flows of sublimation, conduction to the inner layers of the body, stellar radiation absorption and thermal reemission. It gives:

$$
\begin{aligned}
\rho(r) C(r) \frac{\partial T}{\partial t}=F_{\text {incident }}( & -a)-\epsilon \sigma T^{4} \\
& +Q_{\epsilon} \cdot H(T)-K(r, T) \frac{\partial T}{\partial r}
\end{aligned}
$$

where $F_{\text {incident }}$ is the radiation flux received per unit surface, $a$ is the surface Bond albedo, $\epsilon$ is the thermal emissivity and $Q_{\epsilon}$ the sublimation rate at the surface, in moles per second per surface unit. $H(T)$ stands for the latent heat of sublimation.

\subsection{Gas diffusion}

The gas diffusion equation takes the form:

$\frac{1}{R T} \frac{\partial P}{\partial t}=\nabla \cdot(G(r, T, P) \cdot \nabla P)+Q_{\epsilon}$,

where $G$ is the gas diffusion coefficient throughout the porous matrix and $Q_{\epsilon}$ is the source term accounting for the physical processes encountered (sublimation and recondensation).

The way to compute the gas diffusion coefficient $G$ depends on the Knudsen number $K_{\mathrm{n}}$ :

$K_{\mathrm{n}}=\frac{l}{2 R_{\mathrm{p}}}$,

where $l$ is the mean free path, and $R_{\mathrm{p}}$ is the characteristic pore radius. $l$ is given by:

$l=\frac{M}{\sqrt{2} \pi d^{2} \rho \mathcal{N}}$,

where $M$ is the molar mass, $d$ the diameter of the molecule, $\rho$ the density of the gas mixture, and $\mathcal{N}$ is Avogadro's number.

When $K_{\mathrm{n}}>1$, collisions of molecules with the surface of the pores are predominant with respect to moleculemolecule collisions. In this case, kinetic gas theory gives for the gas diffusion coefficient $G$ :

$G_{\text {Knudsen }}=R_{\mathrm{p}} \cdot \sqrt{\frac{\pi}{2 M R T}}$ moles s $^{-1} \mathrm{~m}^{-1} \mathrm{~Pa}^{-1}$.

When $K_{\mathrm{n}}<0.01$, collisions between molecules are predominant, and diffusion is in the viscous mode. In that case, $G$ is a function of the dynamic viscosity and the total pressure. In practice however, it can be easily shown that we are always in Knudsen mode.

The boundary conditions of the gas diffusion equation are, at $r=0$ :

$\frac{\partial P}{\partial r}=0$

and at the surface, as the gas immediately diffuses into space, we can assume that $P=0$. Taking into account that the residual local gas density in this disk is less than a few $10^{6} \mathrm{~cm}^{-2}$, in order to be compatible with the observational limits for hydrogen (Lagrange et al. 1998), we may safely neglect the pressure due to this gas with respect to the saturated vapour pressure, the former being of the order of $\sim 10^{-10} \mathrm{~Pa}$ at most, and the latter one of the order of $0.1 \mathrm{~Pa}$ at $200 \mathrm{~K}$. 


\subsection{Numerical resolution}

The spherical body is subdivided into concentric shells of constant thickness from surface to center. Typically, 500 or 1000 shells are used. The spherical symmetry reduces the problem to a mono-dimensional one, so that the equations may be solved by applying a fourth-order Runge-Kutta method.

As the ice on the surface disperses into space, erosion can occur on the surface of the FEB progenitor. In fact, the $\mathrm{H}_{2} \mathrm{O}$ molecule flow across the surface drags dust grains out of the ice-dust matrix. The detailed simulation of this process is beyond our needs and our present computing capabilities. However we can constrain its effect by taking three extreme models:

- one model where the body is completely icy;

- one model where the dust on the surface erodes with the same rate as ice;

- one model where the dust remains on the surface while the gas flows through it. Surface sublimation then causes the development of an ice-free surface layer.

\subsection{Physical and numerical parameters}

The model nucleus is considered in thermal equilibrium at the beginning of the simulation, i.e., it has a uniform temperature which is computed by considering the equilibrium at the surface between sublimation, stellar radiation absorption and thermal re-emission. By doing it this way, we obtain a good representation of the thermal profile of a body orbiting the star on a circular orbit. The initial gas pressure in the body is the saturated vapor pressure of ice. The initial dust-to-ice mass ratio is equal to 1 . The density of the compact dust material is equal to $b_{\text {dust }}=2120 \mathrm{~kg} \mathrm{~m}^{-3}$, which corresponds to the bulk density of $\mathrm{SiO}_{2}$. The density of ice is $b_{\text {ice }}=1000 \mathrm{~kg} \mathrm{~m}^{-3}$. The initial porosity is $\Pi=0.7$. The pore radius is supposed to be $R_{\mathrm{p}}=10 \mu \mathrm{m}$. All those values have been chosen to mimic those of Solar comet models.

The thermal conductivity of compact crystalline ice has been evaluated by Klinger (1980), by means of laboratory experiments:

$K_{\mathrm{H}_{2} \mathrm{O}}=\frac{567}{T} \mathrm{~W} \mathrm{~m}^{-1} \mathrm{~K}^{-1}$.

The conductivity for compact $\mathrm{SiO}_{2}$ is taken from Van Dusen (1929):

$K_{\mathrm{d}}=1.45 \times\left[1+2.3 \times 10^{-3}(T-273.16)\right] \mathrm{W} \mathrm{m}^{-1} \mathrm{~K}^{-1} \cdot(14)$

This formula is valid in the temperature range $23-373 \mathrm{~K}$, which is sufficient for our needs.

The specific heat for crystalline ice has been fitted by Klinger (1980) from measured data given by Giauque \& Stout (1936):

$C_{\mathrm{p}, \mathrm{H}_{2} \mathrm{O}}=7.49 T+90 \mathrm{~J} \mathrm{~kg}^{-1} \mathrm{~K}^{-1}$.

The specific heat of silica is interpolated from tables given by Sosman (1928).
The specific heat of water vapour is $C_{\mathrm{v}}=$ 27.5 $\mathrm{J} \mathrm{mole}^{-1} \mathrm{~K}^{-1}$. The latent heat of sublimation of pure $\mathrm{H}_{2} \mathrm{O}$ ice is given by Delsemme \& Miller (1971):

$H=51983.9-20.0904 T \mathrm{~J}_{\mathrm{mole}}{ }^{-1}$.

The saturated vapour pressure for ice is taken from Washburn (1928):

$$
\begin{array}{r}
\log P_{\mathrm{S}}(\mathrm{Pa})=\log \left(\frac{101325}{760}\right)-\frac{-2445.5646}{T}+8.2312 \log T \\
-0.01677006 T+1.2051410^{-5} T^{2}-6.757169
\end{array}
$$

for $T>100 \mathrm{~K}$ and

$$
\begin{aligned}
\log P_{\mathrm{S}}(\mathrm{Pa})=\log ( & \left.\frac{101325}{760}\right)-\frac{-2461}{T}+3.857 \log T \\
& -3.4110^{-3} T+4.87510^{-8} / T^{2}
\end{aligned}
$$

for $T<100 \mathrm{~K}$.

Measurements of the optical properties of the solar comet $1 \mathrm{P} /$ Halley have revealed a very dark surface, with an albedo equal to 0.05 (Keller et al. 1987). As this value was obtained from an extrapolation from a large phase angle, an albedo close to 0.1 , which is a good value for a mix of ice and refractory materials, is possible. We have therefore used an albedo of 0.1 and an infrared emissivity of 0.9 .

\section{A pure ice FEB progenitor}

\subsection{Full model}

In this model, ice represents all the matter, and the body simply erodes as the surface sublimates.

Simulations performed at distance of 4 or $8 \mathrm{AU}$ show that an ice ball completely sublimates in a very short time. A body of porosity 0.3 and of radius $1 \mathrm{~km}$ disappears at $4 \mathrm{AU}$ in approximatively 14 years, and at $8 \mathrm{AU}$ in approximatively 180 years. The erosion rate is almost constant, which leads us to the conclusion that the lifetime of an object is proportional to its radius. We also performed simulation with other porosity values: the surface sublimation rate appears to be independent of the porosity. This is a direct outcome of Eq. (1), as the temperature is constant. Hence, the lifetime of the body is proportional to the quantity of matter it contains.

The lifetimes found for those distances show that icy balls are unlikely to survive more than $\sim 10^{6}$ yr at $8 \mathrm{AU}$ or closer, for initial radii up to $10^{4} \mathrm{~km}$. At $8 \mathrm{AU}$, even a $10^{5} \mathrm{~km}$ radius body evaporates within that time span. Such large bodies are unlikely to be sufficiently numerous to produce all the observed features.

\subsection{A simplified model}

The simulations showed that the surface temperature, and therefore the surface sublimation, are constant with time. It is thus justified to compute the equilibrium temperature 


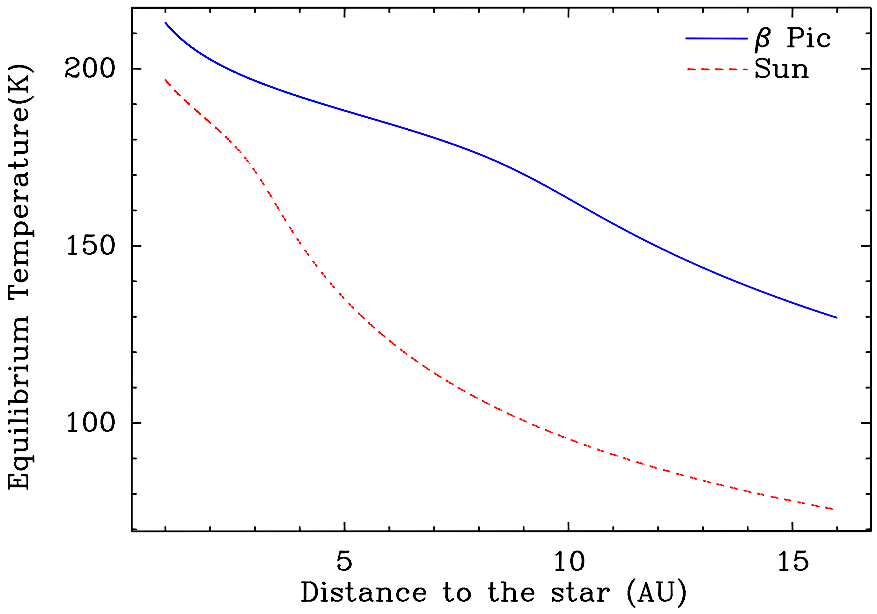

Fig. 1. Equilibrium temperature computed for icy bodies at a given distance from $\beta$ Pic. Similar data in the Solar environment are shown for comparison purposes.

at a given distance from the energy balance, and derive the sublimation rate.

The equilibrium temperature is then computed by resolving the energy balance equation, derived from Eq. (7):

$0=F_{\text {incident }}(1-a)-\epsilon \sigma T^{4}+Q_{\epsilon} H(T)$,

which is solved with a Newton-Raphson algorithm. The sublimation rate on the surface is then:

$Q_{\epsilon}=\frac{p^{s}}{\sqrt{2 \pi M R T_{\mathrm{eq}}}}$.

The decrease of volume of the body per unit time is:

$\frac{\mathrm{d} V}{\mathrm{~d} t}=\frac{Q_{\epsilon}}{M \rho_{\text {ice }}} \cdot 4 \pi r^{2}$

where $\rho_{\text {ice }}$ is the density of the icy matter, taking into account the porosity:

$\rho_{\text {ice }}=b_{\text {ice }}(1-\Pi)$.

The radius $r$ of the body shrinks with the rate:

$\frac{\mathrm{d} r}{\mathrm{~d} t}=\frac{\mathrm{d} r}{\mathrm{~d} V} \frac{\mathrm{d} V}{\mathrm{~d} t}=\frac{1}{4 \pi r^{2}} \frac{Q_{\epsilon}}{M \rho_{\text {ice }}} \cdot 4 \pi r^{2}=\frac{Q_{\epsilon}}{M \rho_{\text {ice }}}$.

As the equilibrium temperature is constant, this rate is constant too, and is independent of the radius. The radius decreases linearly with time. Therefore, the time to fully sublimate the body can be derived exactly:

$t=\frac{r}{\mathrm{~d} r / \mathrm{d} t}=\frac{r M \rho_{\text {ice }}}{Q_{\epsilon}}=\frac{r M b_{\text {ice }}(1-\Pi)}{Q_{\epsilon}}$.

This equation shows that the lifetime of the body is proportional to its radius $r$ and to $1-\Pi$, as was shown by the previous simulations.

Lifetimes and temperatures found by this method are very consistent with our simulations. The results are shown in Figs. 1 and 2.

With this method, we can see precisely that the limit for a lifetime of $10^{6} \mathrm{yr}$ for a one kilometer body is $13.4 \mathrm{AU}$. For a 10 kilometers body it is $12.0 \mathrm{AU}$.

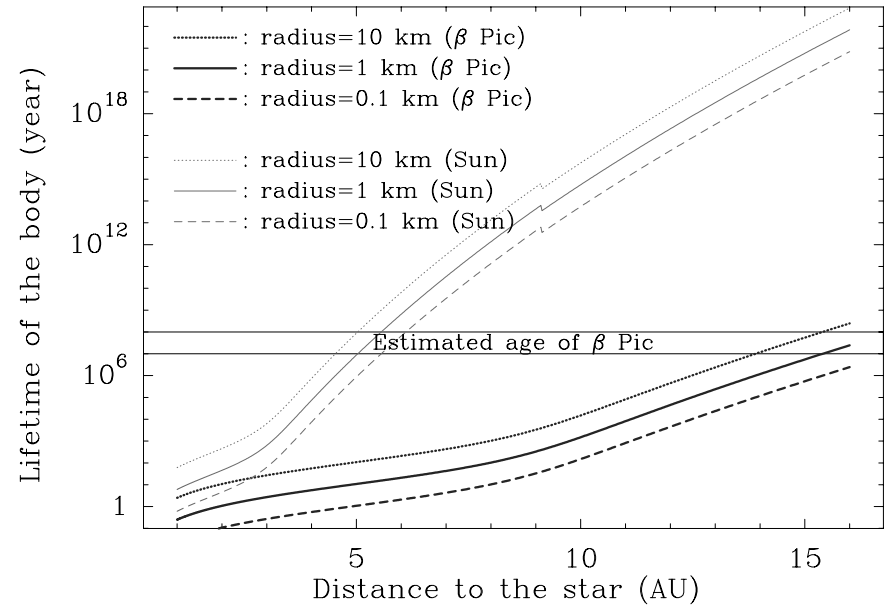

Fig. 2. Lifetime of pure ice bodies for various distances from $\beta$ Pic and the Sun. We can see that at $4 A U$, the nucleus disappears completely in $4.14 \times 10^{8}$ s, i.e. in 13 years, 46 days and 3 hours.

\section{A dust-ice mixed FEB progenitor}

If the body contains some dust, the phenomenon is slightly different. If we focus on the surface, we can see that dust is an obstacle for the sublimation of ice. Following Enzian et al. (1997), we model this, assuming the following form for the $\Sigma$ coefficient in Eq. (1):

$\Sigma=N_{\mathrm{H}_{2} \mathrm{O}} /\left(N_{\mathrm{H}_{2} \mathrm{O}}+N_{\text {dust }}\right)$.

Therefore, for the same temperature, the ice sublimation rate on the surface is smaller. Then, the equilibrium surface temperature is readjusted. The sublimation rate is therefore slightly different.

An important effect is the ejection of dust grains into space, caused by outflow of vapor throughout the surface. This effect induces a segregation between the grains, taking the smaller ones and leaving the bigger ones. Computing the dust ejection rate in term of quantity of matter is therefore very complicated. In order to constrain this effect, we took two extreme models: first, one where the gas outflow takes away all the grains (hereafter referred as Model A), and second, one where all the grains are left where they are (hereafter referred as Model B), any realistic description being assumed to lie somewhere between these two extremes.

\subsection{Model A}

For Model A, the results of the simulations we performed are similar to those obtained with the pure ice model. The ice simply sublimates on the surface and takes away all the dust which lies within. Basically, it behaves as if it were made of a single species of matter with a slightly different sublimation rate.

In this model, the lifetime of a given body is in fact less than in the pure ice case, though still comparable. This may be explained as follows: for the ice itself, the same sublimation rules as described in Sect. 3 apply; 


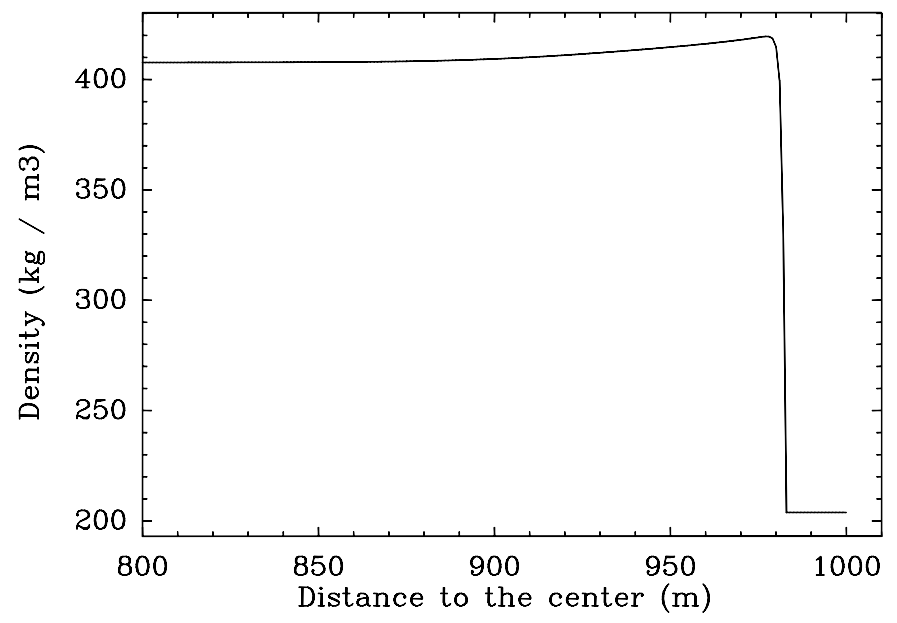

Fig. 3. Density profile of the upper layers of a 200-year old, $1 \mathrm{~km}$ radius body of porosity 0.7 at $8 \mathrm{AU}$. The low density region is the surface layer, which has lost all its ice. The density's pike just below the sublimating layer is due to the recondensation of vapor that has diffused into this region from the adjacent layers, where sublimation occurs.

the sublimation rate of ice at the surface of the body remains thus almost unchanged with respect to the pure ice case, but as the bulk matter constituting the body contains less ice per unit volume, the same ice sublimation rate as before implies a larger rate of decrease of the radius. Hence, in this framework, the lifetime of any FEB progenitor turns out to linearly depend on the icy content of the material constituting the body. A purely icy body of porosity 0.7 disappears within $\sim 115 \mathrm{yr}$, but within only $80 \mathrm{yr}$ if the mass ice/dust ratio is $\sim 1$.

Given this, the simplified model described above still applies, with some minor modifications. The density $\rho_{\text {ice }}$ now reads:

$\rho_{\text {ice }}=\frac{(1-\Pi) b_{\text {ice }}}{1+\frac{b_{\text {ice }}}{b_{\text {dust }}} \lambda}$,

where $b_{\text {ice }}$ and $b_{\text {dust }}$ are the bulk density of, respectively, ice and dust. $\lambda$ is the dust-ice mass ratio. And so, the lifetime of our body is:

$t=\frac{R}{\mathrm{~d} R / \mathrm{d} t}=\frac{R M \rho_{\text {ice }}}{Q_{\epsilon}}=\frac{R M b_{\text {ice }}(1-\Pi)}{Q_{\epsilon}\left(1+\frac{b_{\text {ice }}}{b_{\text {dust }}} \lambda\right)}$.

This expression is in perfect agreement with the results of our simulations, and gives the dependence to the dust-ice mass ratio $\lambda$.

\subsection{Model B}

In the model where all the dust remains in the FEB progenitor, the radius of the body does not shrink. The lifetime of the body itself is in fact infinite, but the lifetime of ice within the body is finite. This is the parameter we want to derive.

The ice simply evaporates as the temperature wave penetrates into the body. This process appears to be much

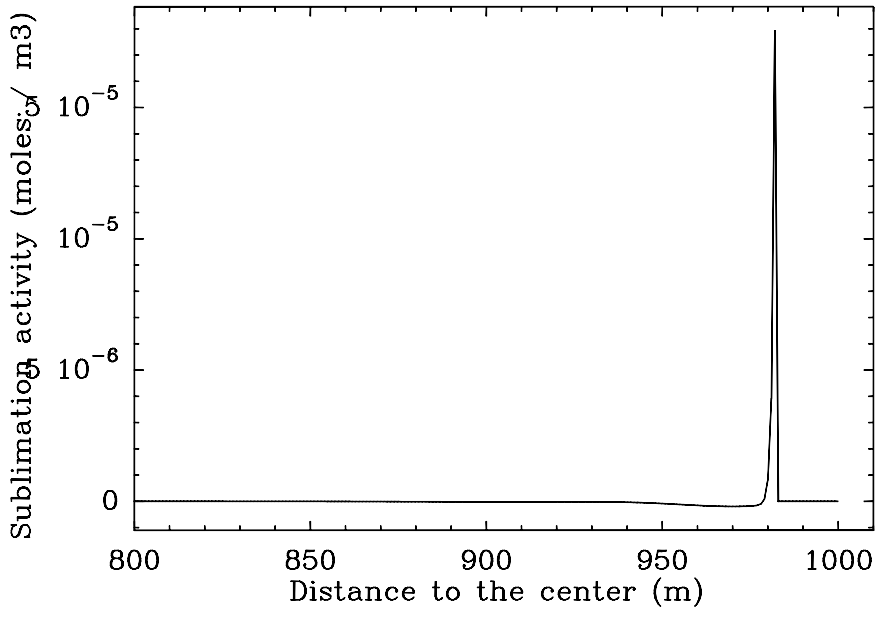

Fig. 4. Sublimation activity in the upper layers, for the same body as in Fig. 3. The sublimation appears to be extremely confined in a very thin region.

slower than the ice evaporation in the pure ice case, and in Model A. Consequently, the lifetime of ice within the body is significantly longer.

Simulations reveal that the internal structure of the body gradually differentiates. We typically find a surface layer where the ice has been entirely evaporated and where only refractory material remains, an intermediate layer where almost all the sublimation process occurs, and a deeper nucleus which preserves its initial state in term of composition, temperature and pressure. This differentiation is illustrated in Figs. 3 and 4 which show the internal structure of a typical body after 200 years of ice sublimation.

As time passes, the intermediate layer slowly becomes deeper, as ice evaporates and diffuses throughout surface layer to disperse into space. Therefore, the body still loses ice, but slower than in the previous model.

Simulations were done for bodies at several distances and the corresponding lifetimes for ice were deduced from results obtained. A tendency has been observed that the lifetime of ice depends quadratically on the radius, for objects larger than a few hundred meters. These results are shown in Fig. 5. While a body of $1 \mathrm{~km}$ radius loses all its ice in a time significantly shorter than the age of $\beta \mathrm{Pic}$, for a semi-major axis less than $12 \mathrm{AU}, 10 \mathrm{~km}$-sized bodies still retain a non-negligible core of ice after 10 millions years, i.e. the extreme lower limit of the age of the star.

Recall that this model gives upper limits of the lifetime of ice in a FEB progenitor. Therefore, it gives lower limits of the depth of the refractory crust.

Beust \& Morbidelli (2000) showed that in order to resist down to truly star-grazing orbits, the FEB progenitors need to be typically 10 or $15 \mathrm{~km}$-sized, rather than $1 \mathrm{~km}$. Therefore, the conclusion is different from that of model A: the quantity of ice in FEB progenitors depends on the time passed in the proximity of the star, and therefore on the age of $\beta$ Pic. For an age of 20 millions years, the depth of the refractory crust of FEB progenitors at $4 \mathrm{AU}$ 


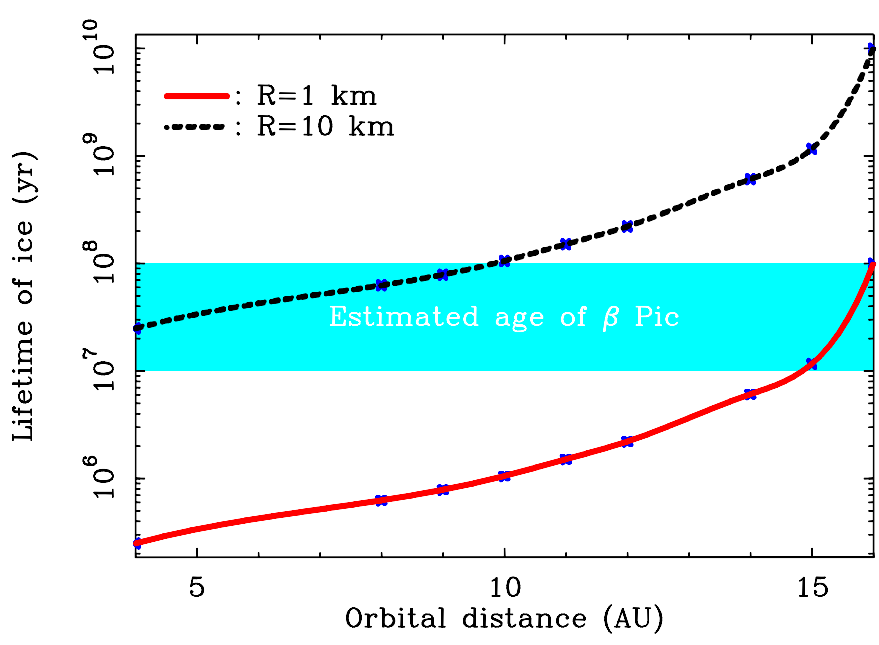

Fig. 5. Time needed to remove all the ice from bodies of porosity 0.7 as a function of their orbital distance to $\beta$ Pic.

is $9 \mathrm{~km}$. For $8 \mathrm{AU}$, it is $5 \mathrm{~km}$. The depth of the refractory crust is shown in Fig. 6 as a function of the semi-major axis, and is parameterized by the age of the star.

\section{Dynamical aspects}

The modeling presented above shows that if the FEB progenitors originate from stellar distances shorter than $\sim 12 \mathrm{AU}$, they may be totally ice-free, or at least contain a deep crust of refractory materials, with an ice-rich core. It is worth discussing at this point this distance from a dynamical point of view.

As mentioned in the Introduction, according to the present model of Beust \& Morbidelli (1996, 2000), the FEBs originate from the $3: 1$ or $4: 1$ mean-motion resonance with a Jovian-like planet orbiting the star on a moderately eccentric orbit $\left(0.05 \lesssim e^{\prime} \lesssim 0.1\right)$. The framework of this dynamics is known as the restricted three body problem, and it simply rescales with the orbital semi-major axis of the planet. The 3:1 and 4:1 mean-motion resonances are located at $\sim 50 \%$ and $\sim 40 \%$ of the planetary semimajor axis $\left(a^{\prime}\right)$ respectively. Therefore, if we want the FEB progenitors to originate from more than $12 \mathrm{AU}$, then the planet must orbit the star at a distance of $\sim 30 \mathrm{AU}$ at least. This fairly large orbital distance immediately raises two problems.

First, as noted above, the dynamical problem rescales with $a^{\prime}$, but not the evaporation limit of dust around the star ( 0.4 AU). Then, for a larger $a^{\prime}$, the same FEB candidates must reach a higher eccentricity to enter the observable FEB regime (i.e., reaching periastron values less than $0.4 \mathrm{AU})$. In their orbital evolution, the resonant bodies undergo a gradual eccentricity increase up to a maximum (close to 1) which depends on their initial orbital configuration. Hence for a larger $a^{\prime}$, a smaller fraction of the resonant bodies are expected to reach a maximum eccentricity that allows them to get into the FEB regime. In other words, we expect less numerous spectral events for the same FEB progenitor population.

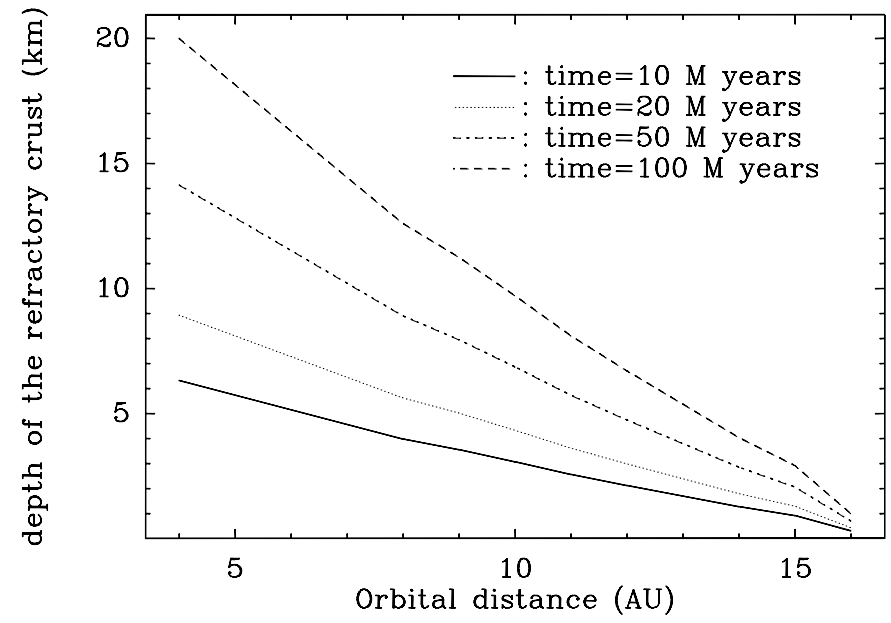

Fig. 6. Depth of the refractory crust for bodies of porosity 0.7 , for different ages of $\beta$ Pic.

In order to illustrate this fact, we carried out numerical simulations of this phenomenon, letting a fixed population trapped in 4:1 resonance evolve under the effects of planetary perturbations, for various values of $a^{\prime}$ (and fixed planetary eccentricity $\left.e^{\prime}=0.07\right)$. In each case, we compute the number of expected FEB spectral events. The framework of the simulation is the same as described in Beust \& Morbidelli (2000). In particular, the integration is performed using the SWIFT package by Levison \& Duncan (1994).

The results for $a^{\prime}=10 \mathrm{AU}$ and $a^{\prime}=40 \mathrm{AU}$ are displayed in Fig. 7. In the latter case, frequency of FEB event appears 16 times less than in the former case. In fact, various tests showed that the FEB frequency simply scales as $a^{\prime-2}$.

From our sample spectral data of $\beta$ Pic gathered over more than 10 years, it has been possible to estimate the average frequency of FEBs events to $\sim 1000$ events per year, with variations by more than a factor 2 between different epochs (see Beust et al. 1996). On the basis of this estimate Beust \& Morbidelli (2000) used similar simulations to derive the local population of the disk around the resonance needed to account for it. They typically derived populations of a few $10^{8}$ bodies per AU. Assuming a classical $r^{-3.5}$ size distribution among the planetesimals, they derived (though with large uncertainty) the planetesimal mass corresponding to such a population, i.e., typically a few tens of Earth masses per AU. Such a mass density in a primordial planetesimal disk is high, but still in agreement with estimates by Weidenschilling (1977) in the primordial Solar System.

These calculations were made assuming $a^{\prime}=10 \mathrm{AU}$. For $a^{\prime}=40 \mathrm{AU}$, the same argument leads to a mass density 4 times larger. The required local disk density necessary to account for the observed FEB frequency turns out to scale as $a^{\prime}$, and not $a^{\prime 2}$ (because the resonance width scales as $\left.a^{\prime}\right)$. Our fixed number of particles is spread over a semimajor axis range which is proportional to $a^{\prime}$. Hence for 
a number of events scaling as $a^{\prime-2}$, the required density scales only as $a^{\prime}$.

For $a^{\prime}$ in the range 30-40 $\mathrm{AU}$, the disk density now falls around $\sim 100 M_{\oplus} \mathrm{AU}^{-1}$, which is no longer realistic. Weidenschilling (1977) derived a primitive surface density scaling as $r^{-3 / 2}$ in the primitive Solar nebula, and so a linear mass density $\propto r^{-1 / 2}$. Assuming this scaling law, if now we need $100 M_{\oplus}$ of planetesimals per $\mathrm{AU}$ at $40 \mathrm{AU}$, then we should expect to have 200 at $10 \mathrm{AU}$. This is unrealistic.

Even if this was possible, a second dynamical constraint makes it difficult to imagine that the perturbing planet lies so far away from the star. When the FEBs are at high eccentricity, their periastron is very small, but their apoastron is close to $\sim 2 a$ if $a$ is their semi-major axis. Hence the FEBs virtually travel across all the disk located inside the orbit of the perturbing planet. If other planets orbit the star closer than the one we are considering, we should expect FEBs to undergo some close encounters with them, with possible ejection from the resonance and thus from the FEB process. In Beust \& Morbidelli (2000), simulations were made assuming the presence of an Earth-sized planet orbiting the star well inside the orbit of the main perturbing planet. They showed that a significant number of bodies were affected, but this did not cause the disappearance of the FEB phenomenon, basically because the planet is small. Moreover, some of the bodies that were diverted from their resonant evolution by the terrestrial planet appeared to still approach the star with another orientation and possibly account for the blueshifted spectral events sometimes observed.

We made similar simulations, now assuming larger planets instead of Earth-sized one. Large inner planets, even down to Uranus-sized planets, turn out to completely abolish the FEB process, almost all bodies undergoing an encounter with the secondary planet. Therefore, an additional requirement of the model is that the planet responsible for the FEB phenomenon via its inner mean-motion resonances must be the innermost giant one. Conversely, outer planets (giant or not) are not excluded, as shown in Beust \& Morbidelli (2000). Now, if we scale our knowledge of the Solar System to the $\beta$ Pic case, even if we take into account that $\beta$ Pic is significantly brighter than the Sun, assuming an innermost giant planet orbiting the star at $40 \mathrm{AU}$ seems less realistic than at $10 \mathrm{AU}$, even if we cannot definitely rule out this possibility.

Finally, even if we cannot severely constrain the semimajor axis of the planet, it is much more likely to be around $10 \mathrm{AU}$ than $40 \mathrm{AU}$. $20 \mathrm{AU}$ may be regarded as an upper limit. Consequently the FEB progenitors cannot be completely icy. If they contain ice, it is in the form of an ice-rich core. They may look like asteroids with an icy nucleus rather than a comet.

\section{Consequence for the FEB evaporation model}

The probable absence of ice, and thus of volatile material, in the upper layers of the FEB progenitors leads to a re-

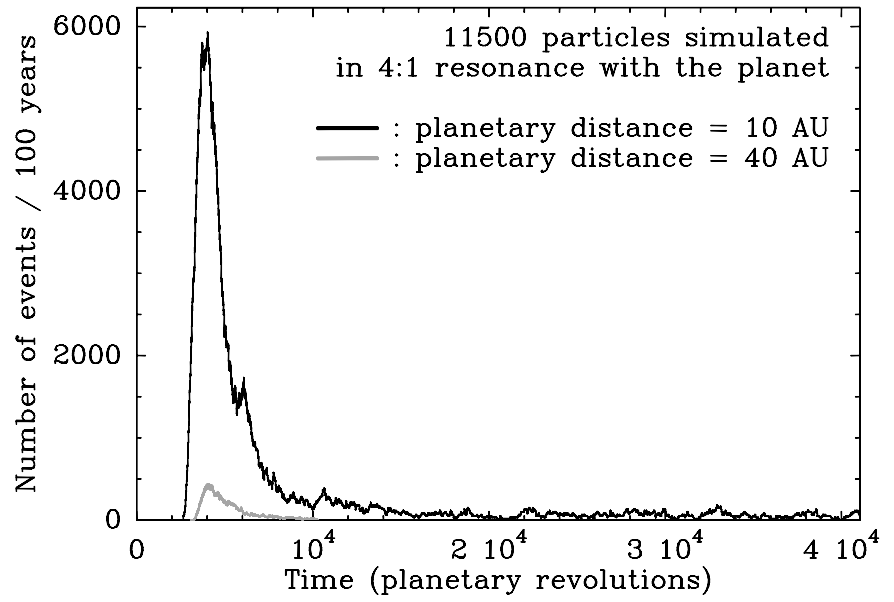

Fig. 7. Compared simulation outputs of the efficiency of the $4: 1$ resonance for generating FEBs, for a planet located at $10 \mathrm{AU}$, and at $40 \mathrm{AU}$. In each case, the same number of bodies (1500) was initially put into the $4: 1$ resonance and their motion numerically integrated. The curves display the number of FEB spectral events to be expected as a function of the time, for both cases. The mechanism appears considerably more efficient in the $10 \mathrm{AU}$ case.

vision of the FEB evaporation model that was assumed thus far. This statement is reinforced by the fact that the results of our model B gives a lower limit to the sublimation rate of ice in the progenitors (and thus an upper limit to the survival time of ice), while model A gives an upper limit (and so a lower limit to the lifetime of ice). Moreover, the ice core which could still be present today in the $10 \mathrm{~km}$-sized (or more) FEB progenitors is likely to start evaporating more quickly as soon as the body starts to increase its eccentricity and make closer approaches to the star. It is even not clear whether ice will still be present when the periastron distance of the body reaches the dust evaporation limit $(\sim 0.4 \mathrm{AU})$.

Only when it reaches this dust evaporation zone may a given FEB crossing the line of sight close to periastron be observed in absorption. However, it can be observed only if it develops around it a large and dense enough coma of metallic material. The coma needs to be large in order to mask a significant fraction of the stellar surface, and it also needs to be dense enough to be optically thick in the spectral lines observed. Note that both conditions are required. The second one is fulfilled if the FEBs evaporates at a high enough rate. Previous studies (see e.g. Beust et al. 1996) have shown that the required rates are typically $\sim 3 \times 10^{7} \mathrm{~kg} \mathrm{~s}^{-1}$ within one order of magnitude. The first condition is fulfilled only if the material escaping from the nucleus is partly made of species undergoing very little radiation pressure. Most of the spectral lines exhibiting FEB variable components concern metallic species that undergo a high radiation pressure (Lagrange et al. 1998). When released by the body, these ions are immediately subject to the stellar radiation pressure, so that if nothing else is able to retain them, they are blown away and the resulting coma is too thin to allow a detection. If the 
ions encounter a dense enough medium that does not suffer a strong radiation pressure, then collisions can prevent them from being too quickly blown away, and a large coma forms (Beust et al. 1996).

As mentioned in the Introduction, volatiles (dissociated water, $\mathrm{CO}$ ) can actually retain the ions, as they suffer very little radiation pressure (Lagrange et al. 1998). If the bodies are made of a mixture of refractory material and ices, then the evaporation of ices provides the needed volatile medium. This is the framework of the simulations presented thus far (Beust et al. 1996, 1998).

Now if the FEB progenitors contain only a small amount of ice (and maybe no ice at all), then their ability to be observed when they reach the FEB regime needs to be questioned. Volatiles are needed as they are abundant species that do not suffer a strong radiation pressure from the star. However, in the bulk refractory material constituting the FEBs (basically carbonaceous material, silicates and oxides in proportions that may vary), there are species that undergo a very small radiation pressure.

The radiation pressure is usually referred to by its constant ratio $\beta$ to stellar gravity. $\beta$ is typical for a given species, and results from the contribution of all its resonance spectral lines. Basically, all metallic species have $\beta$ values larger than 1 in the environment of $\beta$ Pic (see a list of $\beta$ values in Lagrange et al. 1998), and are thus strongly repelled by the star. However, C I and Si II have small $\beta$ values $(0.095$ and 0.15$)$. Neutral oxygen $(\mathrm{O} I)$ and $\mathrm{CO}$, which are not listed in the table of Lagrange et al. (1998) have even smaller $\beta$ values, as their main spectral lines do not fall in spectral ranges where the flux of $\beta$ Pic is important. In standard models or cometary dust composition (see e.g Jeßberger et al. 1987, 1991), these species are fairly abundant with respect to other ones. Note that circumstellar components of $\mathrm{C}$ I and $\mathrm{CO}$ have been observed in the spectrum of $\beta$ Pic (Vidal-Madjar et al. 1994), showing that these species are actually present.

The species listed above that do not suffer a strong radiation pressure may then be abundant enough to retain the other metallic species around a FEB nucleus, and thus allow it to develop an observable coma. The model used thus far including volatiles must then be revised, volatiles being at least partly replaced by these species. This issue can however not be straightforwardly investigated as a small modification of the simulation code dedicated to FEB evaporation described in Beust et al. (1990, 1996, 1998). The dynamics of all the species constituting the evaporating dust must now be computed simultaneously, and not separately as before. This adjustment is presently under development and will be presented in a future work.

\section{Conclusion}

In order to constrain the chemical composition of the FEB progenitors, we have developed a model of evolution of those bodies on a circular orbit at a given semi-major axis. It is an exploratory model, based on previous monodimensional models which were built for solar comets.
In order to answer the question of the presence of volatiles in those progenitors, which is related to the modeling of the transient spectral event, we used three exploratory models, the last two giving us respectively an upper and a lower limit of the sublimation rate of ice.

Finally, it turns out that demanding the FEB progenitors to be icy in a major part of their volume is probably an unrealistic picture, although it cannot be definitely ruled out. They are probably composed of mixed dust-ice nuclei surrounded by a thick refractory crust. The radius of the icy nucleus depends on the radius of the objects, their semi-major axis and the age of $\beta$ Pictoris. We feel that this will lead to a revision of the physics of the evaporation of FEBs.

\section{References}

Artymowicz, P. 1997, Ann. Rev. Earth Planet. Sci., 25, 175

Barrado y Navascués, D., Stauffer, J. R., Song, I., \& Caillault, J.-P. 1999, ApJ, 520, L123

Beust, H., Lagrange-Henri, A.-M., Vidal-Madjar, A., \& Ferlet, R. 1990, A\&A, 236, 202

Beust, H., Lagrange, A.-M., Plazy, F., \& Mouillet, D. 1996, A\&A, 310, 181

Beust, H., \& Morbidelli, A. 1996, Icarus, 120, 358

Beust, H., Lagrange, A.-M., Crawford, I. A., et al. 1998, A\&A, 338,1015

Beust, H., \& Morbidelli, A. 2000, Icarus, 143, 170

Brunini, A., \& Benvenuto, O. G. 1996, MNRAS, 283, L84

Chapman, S., \& Cowling, T. G. 1960, The Mathematical Theory of Non-Uniform Gases (Cambridge University Press), London

Crifo, F., Vidal-Madjar, A., Lallement, R., Ferlet, R., \& Gerbaldi, M. 1997, A\&A, 320, L29

Delsemme, A. H., \& Miller, D. C. 1971, Planet. Space. Sci., 19, 1229

Enzian, A., Cabot, H., \& Klinger, J. 1997, A\&A, 319, 995

Espinasse, S., Klinger, J., Ritz, C., \& Schmidt, B. 1989, A method of estimating temperature profiles and chemical differentiation in the near surface of porous comet nuclei - First results for comet P/Churyumov-Gerasimenko, Physics and Mechanics of Cometary Materials, ed. J. Hunt, \& T. D. Guyenne, ESA SP-302, 185

Espinasse, S., Klinger, J., Ritz, C., \& Schmidt, B. 1991, Icarus, 92,350

Fanale, F. P., \& Salvail, J. R. 1987, Icarus, 72, 535

Giauque, W. F., \& Stout, J. W. 1936, J. Am. Chem. Soc., 58, 1144

Grinin, V., Natta, A., \& Tambovtseva, L. 1996, A\&A, 313, 857

Jeßberger, E. K., Kissel, J., Fechtig, H., \& Krüger, F. R. 1987, On the Average Chemical Composition of Cometary Dust, Physical Processes in Comets, Stars, and Active Galaxies, ed. W. Hillebrandt, E. Meyer-Hofmeister, \& H.-C. Thomas (Springer), 26 
Jeßberger, E. K., \& Kissel, J. 1991, Chemical Properties of Cometary Dust and a Note on Carbon Isotopes, Comets in the Post-Halley Era, ed. R. L. Newburn, J. Rahe, \& M. Neugebauer (Kluwer Academic Publishers), 1075

Keller, H. U., Delamere, W. A., Huebner, W. F., et al. 1987, A\&A, 187, 807

Klinger, J. 1980, Science, 209, 271

Lagrange, A.-M., Plazy, F., Beust, H., et al. 1996, A\&A, 310, 547

Lagrange, A.-M., Beust, H., Mouillet, D., et al. 1998, A\&A, 330, 1091

Lagrange, A.-M., Backman, D. E., \& Artymowicz, P. 2000, Planetary Material around Main Sequence Stars Protostars and Planets IV, ed. V. Mannings, A. P. Boss, \& S. S. Russell (University of Arizona Press, Tucson), in press

Lanz, T., Heap, S. R., \& Hubeny, I. 1995, ApJ, 447, L41

Levison, H. F., \& Duncan, M. J. 1994, Icarus, 108, 18

Paresce, F. 1991, A\&A, 247, L25

Smith, B., \& Terrile, R. 1984, Science, 226, 1421
Spohn, T., Seiferlin, K., \& Benkhoff, J. 1989, Thermal conductivities and diffusivities of porous ice samples at low pressures and temperatures and possible modes of heat transfer in near surface layers of comets, Physics and Mechanics of Cometary Materials, ed. J. Hunt, \& T. D. Guyenne, ESA SP-302, 77

Sosman, R. B. 1929, International Critical Tables, vol. V, 105

Tambovtseva, L. V., \& Shestakova, L. I. 1999, Planet. Space Sci., 47, 319

Tancredi, G., Rickman, H., \& Greenberg, J. M. 1994, A\&A, 286,659

Van Dusen, M. S. 1929, International Critical Tables, vol. V, 216

Vidal-Madjar, A., Lagrange-Henri, A.-M., Feldman, P. D., et al. 1994, A\&A, 290, 245

Vidal-Madjar, A., Lecavelier des Etangs, A., \& Ferlet, R. 1998, Planet. Space Sci., 46, 629

Washburn, E. 1928, International Critical Tables, vol. III, 210

Weidenschilling, S. J. 1977, Astr. Sp. Sci., 51, 153 